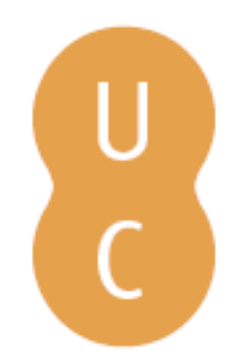

\title{
nombalina
}

\section{L'Europe: allegorie geographique et ideologie imperiale au XIVe. siecle en France}

\author{
Autor(es): Buron, Emmanuel
}

Publicado por: Imprensa da Universidade de Coimbra

URL

persistente: URI:http://hdl.handle.net/10316.2/38958

DOI: $\quad$ DOI:http://dx.doi.org/10.14195/978-989-26-0395-7_12

Accessed : $\quad$ 26-Apr-2023 04:56:06

A navegação consulta e descarregamento dos títulos inseridos nas Bibliotecas Digitais UC Digitalis, UC Pombalina e UC Impactum, pressupõem a aceitação plena e sem reservas dos Termos e Condições de Uso destas Bibliotecas Digitais, disponíveis em https://digitalis.uc.pt/pt-pt/termos.

Conforme exposto nos referidos Termos e Condições de Uso, o descarregamento de títulos de acesso restrito requer uma licença válida de autorização devendo o utilizador aceder ao(s) documento(s) a partir de um endereço de IP da instituição detentora da supramencionada licença.

Ao utilizador é apenas permitido o descarregamento para uso pessoal, pelo que o emprego do(s) título(s) descarregado(s) para outro fim, designadamente comercial, carece de autorização do respetivo autor ou editor da obra.

Na medida em que todas as obras da UC Digitalis se encontram protegidas pelo Código do Direito de Autor e Direitos Conexos e demais legislação aplicável, toda a cópia, parcial ou total, deste documento, nos casos em que é legalmente admitida, deverá conter ou fazer-se acompanhar por este aviso.

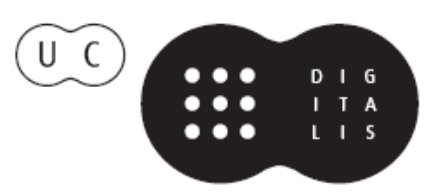


Nair de Nazaré Castro Soares

Santiago López Moreda

Coordenação

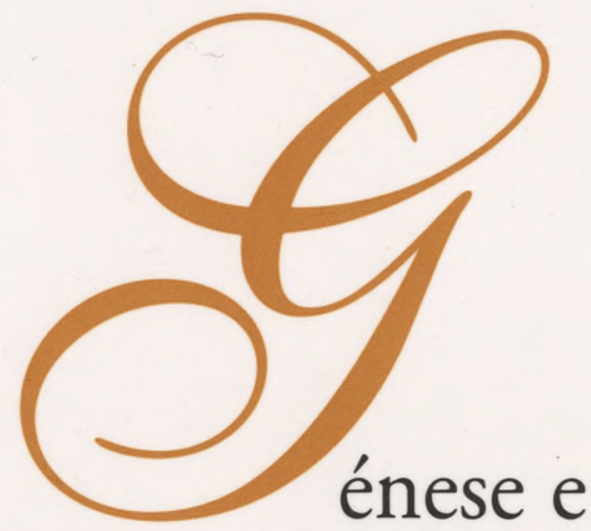

Consolidação da Ideia de Europa

Vol. IV

Idade Média e Renascimento

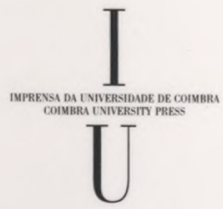

- COIMBra 2009 


\title{
L'EUROPE: ALLEGORIE GEOGRAPHIQUE ET IDEOLOGIE IMPERIALE AU XVI' ${ }^{\mathrm{e}}$. SIECLE EN FRANCE ${ }^{1}$
}

\author{
EMMANUEL BURON \\ (CELAM - Université de Rennes 2)
}

\begin{abstract}
Analyser la genèse de l'idée européenne revient à interroger la puissance qu'ont les mots de susciter des idéaux politiques. En effet, le nom d'Europe désigne un continent bien avant de désigner un quelconque projet collectif: il découpe une zone dans l'espace continu de la planète, sans postuler d'autre unité que la contiguïté entre les territoires qu'il désigne (peut-être trouve-t-il aussi une évidence illusoire dans la position de prétendues "frontières naturelles", l'océan atlantique à un bord, l'Oural à l'autre). Toutefois, l'esprit humain a horreur de l'arbitraire, et, par un réflexe réaliste, il veut croire fondés les noms grâce auxquels il découpe le réel, quitte à construire leur référent. La seule existence du nom "Europe" ouvrait donc la possibilité d'un projet politique propre à lui donner consistance. "L'Europe, c'est un nom flottant et qui pendant longtemps n'a pas su sur quelles réalités exactement se poser", écrit Lucien Febvre dans un beau livre sur lequel nous aurons à revenir ${ }^{2}$ : c'est-à-dire que l'Europe a pris consistance quand le nom a étendu sa pertinence hors du domaine géographique qui a toujours été le sien, et qu'il a reçu un signifié politique. En d'autres termes, l'idée européenne naît d'une glissement sémantique; à son origine, on trouve une figure de rhétorique complexe, qui tient de la métonymie (projection sur des états de l'homogénéité qui caractérise l'espace dans lequel ils se trouvent) et de la métaphore (transfert de la pertinence d'un mot de la géographie vers la politique). L'examen de la notion d'Europe au $\mathrm{XVI}^{\mathrm{e}}$ s., principalement en France, permettra de vérifier ces remarques préalables. Nous verrons que si le nom se diffuse alors comme notion géographique,
\end{abstract}

${ }^{1}$ Cet article constitue une version très largement remaniée d'une étude antérieure: "L'Europe et l'Empire au XVI ${ }^{\mathrm{e}}$ s.", parue dans G. Baudelle et C. Guy, Le Projet européen. Histoire, enjeux, prospective, Rennes, Presses Universitaires de Rennes, 2004, p. 37-47.

${ }^{2}$ L. Febvre, L'Europe. Genèse d'une civilisation, Paris, Perrin, 1999, p. 279. 
il ne fait l'objet d'aucun investissement politique, du moins dans les discours qui visent directement à rendre compte des institutions humaines, celui du mémorialiste ou celui de l'historien par exemple. C'est en revanche dans les représentations picturales ou poétiques que la notion d'Europe se charge peu à peu d'une signification politique. C'est alors par le biais de l'allégorie que s'opère le rapprochement entre l'idée d'Europe et un projet politique (impérial en l'occurrence). Utile rappel pour notre époque, qui conçoit la politique comme une technocratie libérale: pour qu'un grand projet puisse prendre consistance, la politique doit s'appuyer sur une fiction poétique susceptible de lui donner un sens.

Jusqu'au $\mathrm{XV}^{\mathrm{e}}$ siècle environ, le nom d'Europe ne désigne qu'un espace géographique, et il reste d'une diffusion très restreinte: il ne concerne guère que les géographes. Depuis l'Antiquité, le terme est en effet utilisé dans les descriptions du monde: avec l'Afrique et l'Asie, le mot désigne une des trois parties du monde. Pendant tout le Moyen-Âge, il n'existe guère qu'en latin, et il est d'usage savant et spécialisé. Les choses changent au cours du $X V^{e} s$, avec la redécouverte de la Géographie de Ptolémée. En 1452, les Turcs prennent Byzance, et un certain nombre de lettrés fuient cette ville pour l'Italie, emportant avec eux leur connaissance du grec, langue dont la maîtrise était perdue en Europe, et un certain nombre de manuscrits dans cette langue. Manuel Chrysoloras, un de ces lettrés exilés, apporta ainsi à Florence le texte de la géographie de Ptolémée, auparavant connu par des traductions latines réalisées par des savants arabes. Jacopo Angelo, un humaniste florentin, retraduisit directement du grec en latin l'ouvrage ancien, qui connut à partir de là un succès extraordinaire. De nombreuses éditions imprimées se succèderont tout au long du $\mathrm{XVI}^{\mathrm{e}}$ s., mais dès le $\mathrm{XV}^{\mathrm{e}}$ s., de nombreuses éditions manuscrites verront le jour. L'ouvrage est généralement illustré de 27 cartes, soit 1 carte générale du monde et 26 cartes "régionales": 10 pour l'Europe; 12 pour l'Asie; 4 pour l'Afrique. Ces cartes sont éventuellement surmontées d'indication telle "Prima Europa Tabula" ${ }^{3}$. La qualité extraordinaire des manuscrits prouve que l'ouvrage a eu du succès auprès d'un public aristocratique: la géographie gagne alors une place dans la culture des élites politiques du temps, et celles-ci apprennent à se penser dans un cadre géographique mondial, et à dénommer "Europe" la partie du monde où ils vivent. Ainsi Philippe de Commynes, qui dicte ses mémoires à la fin du $X^{\mathrm{e}} \mathrm{s}$. (et sur l'exemple de qui nous reviendrons bientôt): il était chargé du versant italien de la diplomatie de Louis XI, et il

\footnotetext{
${ }^{3}$ Sur la redécouverte de Ptolémée, voir N. Broc, La Géographie de la Renaissance, Paris, C.T.H.S., 1986, ch. 1 et La Géographie de Ptolémée, Arcueil, Anthèse, 1998 (éd. des planches du manuscrit Parisinus latinus 10764 de la BNF, présenté par G. Aujac).
} 
entretenait des rapports privilégiés avec Florence ${ }^{4}$, ville où est arrivé Chysoloras, où Angelo a traduit Ptolémée, et qui, est ainsi devenue, pour tout le $\mathrm{XV}^{\mathrm{e}} \mathrm{s}$., un des grands foyers européens pour le développement de la géographie. Commynes n'a donc eu aucun mal à connaître Ptolémée, et dans le texte de ses Mémoires, il distingue comme lui trois parties du monde: Europe, Afrique, Asie. Toutefois, le découpage d'un espace en zones désignées par un nom ne crée pas pour autant un sentiment d'appartenance politique, et encore moins une identité culturelle. Pour nombre de Français du XVI ${ }^{\mathrm{e}}$ siècle, beaucoup de pays d'Europe demeuraient inconnus; quant aux voisins, ils incarnaient plutôt l'ennemi que le partenaire: quelle consistance accorder au sentiment européen, quand François I ${ }^{\mathrm{er}}$ cultive l'alliance turque dans l'espoir d'étouffer l'Espagne ? Sans doute cette alliance faisait-elle grincer des dents à certains, mais c'était au nom d'une conscience chrétienne, et non européenne. L'appartenance à un même espace géographique ne crée nulle solidarité politique.

Pour le vérifier, arrêtons nous sur un texte qui a déjà retenu l'attention de Lucien Febvre. En 1944-1945, cet éminent historien consacrait son cours du Collège de France à une histoire de l'idée d'Europe ${ }^{5}$. Il y constatait l'ancienneté du nom, et son souci était de dégager la manière dont ce nom en était venu à désigner une "civilisation", celle qui s'est imposée à la place de l'Empire Romain et de la Chrétienté médiévale. Il associait ainsi à l'Europe l'idéal d'un concert des nations, qui aurait peu à peu éliminé le projet de domination universelle, et c'est chez Philippe de Commynes qu'il pensait trouver la première émergence de cette idée. Il décèle chez cet auteur l'orgueil d'être européen, orgueil qui serait à la fois celui d'un chrétien par rapport aux non-chrétiens, et celui d'un occidental par rapport à l'Asie, ancien foyer de la culture en voie d'être dépassé par le progrès de l'Occident ${ }^{6}$. Il faut cependant relire le texte sur lequel s'appuie cette analyse, et le suivre dans sa logique. Commynes énonce d'abord une loi de la Providence, qui, pour limiter la puissance qu'un état pourrait atteindre, place immédiatement à côté de lui un état adversaire, de manière à ce que leur rivalité, et même leurs guerres, les brident mutuellement.

Au fort, il me semble que Dieu n'a creé nulle chose en ce monde, ny hommes ni bestes, à qui il n'ait faict quelque chose son contraire, pour le tenir en humilité et en craincte.

\footnotetext{
${ }^{4}$ Voir J. Blanchard, Commynes et les Italiens, Paris, Klincksieck, 1993

${ }^{5}$ L. Febvre, op. cit.

${ }^{6}$ «Commynes, et cette fois, nous sommes en présence, vraiment d'une Europe et d'un Européen à la fois, d'un Européen fier de l'être, d'un Européen qui considère que d'être Européen, cela suffit, cela lui suffit ; bien plus que d'être européen, c'est une supériorité qui permet à un homme intelligent et cultivé de mépriser tout ce qui n'est pas européen, de traiter de haut et de loin les parties d'Asie et d'Afrique, comme il dit», ibid., p. 178.
} 
Ce principe est d'abord vérifié sur l'exemple de Gand, qui résiste au pouvoir du duc de Bourgogne sur les Flandres, puis par la rivalité des Français avec les Anglais, des Anglais avec les Ecossais, des états d'Italie entre eux, etc. C'est finalement un tableau des guerres entre pays voisins que dresse Commynes, avant de conclure:

Je n'ay parlé que de Europpe, car je ne suis point informé des deux aultres pars, comme d'Asie et d'Affricque; mais bien orrons nous dire qu'ilz ont guerres et divisions comme nous, et encores plus meschamment, car j'ay sceu en ceste Affricque plusieurs lieux où ilz se vendent les ungs les aultres aux chrestiens; et appert par les Portingallois qui maint esclave en ont eu et ont [tous] les jours. Et par ce moyen je doubte que ne les devons point trop [reprocher] aux Sarrasins, et qu'il y a des parties à la chrestienté qui en font autant; mais ilz sont situés soubz le pouvoir du Turc, ou fort voisins, comme en aulcune partie de la Grece?

Malgré la faiblesse de son information, Commynes estime donc que la même loi de division joue en Afrique et en Asie, qui "ont guerres et divisions comme nous". L'esclavage lui en fournit une preuve: puisqu'il y a des Africains pour vendre d'autres Africains aux Portugais, c'est qu'un principe de rivalité entre proches joue en Afrique comme en Europe. Sans doute Commynes tendil à identifier l'esclavage à des mœurs exotiques, mais en dépit de ses réticences, il admet qu'on le rencontre dans toutes les parties du monde, et qu'il constitue un avatar d'une même loi divine, qui se manifeste en Europe sous forme de guerres. Les formes changent, mais l'homme est partout un loup pour son voisin. Tout le texte repose sur l'idée que nous ne sommes ni meilleurs, ni mieux lotis. Nous sommes loin du sentiment de supériorité qu'y trouvait Lucien Febvre: c'est bien plutôt le sentiment d'une égalité des peuples devant la providence qui domine. Quant à la conscience d'une unité de l'Europe, il serait paradoxal de la chercher dans ce tableau d'une Europe à feu et à sang, et qui, par décret divin, ne peut pas connaître de paix.

Febvre surinterprète donc le texte de Commynes, mais il avait d'excellentes raisons pour le faire, et son erreur est en l'occurrence louable, puisqu'il l'a présentée lors d'un cours au collège de France en 1944-1945, pour arracher l'idée européenne à l'idéologie nazie qui s'en était alors emparée ${ }^{8}$ : c'était en somme une nécessité politique que de lui trouver un fondateur à l'aube des temps modernes. Un acte de résistance intellectuelle. Toutefois, en dépit de cette identification d'une origine au $\mathrm{XV}^{\mathrm{e}} \mathrm{s}$., Febvre doit constater que le nom

${ }^{7}$ P. de Commynes, Mémoires, livre V, ch. XVIII, éd. Ph. Contamine, Imprimerie Nationale, 1994 , p. $354-356$

${ }^{8}$ Voir la préface de M. Ferro à L. Febvre, op. cit. 
d'Europe ne fait pas florès au $\mathrm{XVI}^{\mathrm{e}}$ s., et ce constat suffit à jeter rétrospectivement le doute sur la lecture qu'il propose de Commynes: comment le mémorialiste aurait-il fait usage incidemment, comme s'il s'agissait d'une évidence, d'une idée aussi neuve et qui ne prendra véritablement son essor que plus d'un siècle après ? Toujours est-il que si Lucien Febvre relève de nombreuses occurrences du nom "Europe" sous la plume des poètes ${ }^{9}-j^{\prime} y$ reviendrai -, il n'en trouve guère sous la plume des grands intellectuels européens ou des grands penseurs politiques postérieurs à Commynes, comme Erasme, Thomas More, Bodin. De même, on en trouve rarement aussi dans les récits de voyage ou chez les historiens. En général, tous ces auteurs usent de périphrases, ou énumèrent les états dont ils parlent, mais ils ne disposent pas d'un terme collectif d'usage politique $^{10}$. Dans la réflexion historique et politique du $\mathrm{XVI}^{\mathrm{e}}$ siècle, l'Europe ne s'impose pas comme un niveau significatif. Même si elle adopte des formes profondément renouvelées, l'Histoire se partage, comme au Moyen$\hat{A} g e$, entre une histoire nationale et une histoire universelle, ce qui suggère que les esprits politiques les plus clairvoyants ne distinguaient guère d'intermédiaire entre le niveau du pays et celui du monde. L'Europe ne crée ni un devoir de sympathie, ni même la conscience d'une solidarité particulière entre les pays du continent.

Pour le vérifier, arrêtons nous sur un opuscule de Louis Le Roy, publié à Paris chez Federic Morel en 1567, les Considérations sur l'histoire françoise et l'universelle de ce temps. Le Roy tient une place importante dans la pensée politique et historique de la deuxième moitié du XVI ${ }^{\mathrm{e}} \mathrm{s}$. Né vers 1510 à Coutances en Normandie, il se rend à Paris vers 1530 pour apprendre le grec, puis à Toulouse, pour suivre des cours de droit. De retour à Paris en 1540, il publie en latin une vie de Guillaume Budé, essaie sans succès une carrière à la cour, et se consacre à la traduction des grands auteurs grecs (Platon, Aristote, Xénophon, Isocrate, Démosthène) en français. Ce combat pour une prose philosophique en langue vulgaire lui vaudra l'estime des poètes de son temps. Ses choix de traduction révèlent un intérêt marqué pour les questions de politique, qu'il développera par une série d'opuscules de circonstance pendant les guerres de religion et par la traduction des Politiques d'Aristote. En 1572, Le Roy de-

9 Febvre, op. cit., p. 189-192 et 196-198. Pour expliquer cette présence du mot chez les poètes et les hommes de lettres, Febvre invoque d'abord l'imitation de l'Antiquité, qui a usé du mot ; puis la découverte du Nouveau Monde, qui a fait prendre conscience de soi à l'Ancien Monde, et l'a obligé à forger un mot pour se désigner ; enfin l'école latine, qui contribuait à forger une langue commune au niveau européen, et à diffuser une même culture

${ }^{10}$ Febvre, op.cit., p. 192-196 et 201-202. 
vient professeur de grec au Collège Royal; en 1575, il publie son grand ouvrage, De la Vicissitude et variété des choses en l'univers, où il développe sa philosophie de l'histoire, et meurt deux ans après ${ }^{11}$. Le court texte de 1567 qui nous intéresse esquisse un projet historiographique de grande ampleur, pour lequel Le Roy espère obtenir l'approbation de Catherine de Médicis, à qui il dédie la plaquette. Pour obtenir l'appui de la reine, la stratégie de l'humaniste peut sembler assez périlleuse, car il commence par récuser la tâche qu'elle lui avait confiée, et qui consistait à réviser les annales et chroniques de France: "Car ce n'est petite entreprinse de vouloir redresser Annales tant mal digerees que sont celles de France" ${ }^{12}$. Pour Le Roy, ce texte constitue un ramas de fadaises impossible à raccommoder, sauf à tout réécrire. L'humaniste présente à la reine un projet historiographique alternatif: écrire l'histoire des dix dernières années écoulées, depuis la mort d'Henri II jusqu'au moment de la rédaction. L'auteur compense ce qu'une telle histoire perd en durée en élargissant considérablement son champ de vision: cette histoire du temps présent ne doit pas être seulement une histoire française, mais une histoire universelle (d'où le titre) dépassant la simple chronique des faits du royaume pour interroger la cohérence des événements affectant le monde à un moment déterminé. Contre une histoire nationale, qui interroge diachroniquement la cohérence des événements dans un cadre géographique limité, l'histoire universelle qu'envisage Le Roy interroge la cohérence synchronique des événements dans un cadre géographique très large. Un tel renversement de perspective renvoie à l'intuition de Le Roy d'une première mondialisation des relations internationales. Si le royaume de France constitue toujours le centre de son intérêt, il a conscience que l'importance de celui-ci se mesure au fait que l'équilibre du monde dépend de sa ligne de conduite.

LE ROYAUME de France situé au milieu d'Occident, entre l'Ocean et la mer mediterranee, plein de richesses, armes, hommes nobles et vaillants, chevaux, navires, est de telle grandeur et puissance, que les voisins disent en dependre la paix ou la guerre de la Chretienté. Et qu'il ne se peut remuer sans esmouvoir le reste du monde, soit que guerroyons entre nous, ou contre les estrangers, à cause des considerations, alliances, intelligences et correspondances qu'ont les François avecques eulx. ${ }^{13}$

"Sur Louis Le Roy, voir H. Becker, Un Humaniste au XVT s. Loys le Roy (Ludocius regius) de Coutances, Paris, Lecène, Oudin et $\mathrm{C}^{\mathrm{ie}}, 1896 \mathrm{et} \mathrm{W}$. Gundersheimer, The Life and works of Louis Le Roy, Genève, Droz, 1966.

${ }^{12}$ L. Le Roy, Considérations sur l'histoire françoise et l'universelle de ce temps, Paris, F. Morel, 1567, p. $2 \mathrm{v}^{\circ}$.

${ }^{13}$ Ibid, f. 7 r. 
Pour illustrer cette affirmation, Le Roy expose alors brièvement comment tous les pays environnants ont été amenés à prendre parti pour un camp ou pour l'autre, lors des premières guerres de religion en France ou, quelques années auparavant, lors des guerres entre la France et l'Espagne. Il ne nomme alors que des pays d'Europe, laissant un instant penser qu'il n'envisage de géopolitique qu'au niveau de ce continent, mais très vite il élargit ce cadre.

Quand le Turc tourne ses forces vers la Hongrie et l'Autriche, menant avec ses gens grosses troupes de Tartares, il fait paix ou tresves avec le Sophi, ou luy suscite d'autres ennemis pour l'amuser: $\mathrm{Si}$ au contraire il marche vers Orient, il appaise ou remet ses differents de par dela. [...] Les Castillans et Portugallois ont les Mollucques contentieuses entre eux, et s'efforcent empescher les François et Anglois d'aller à la Floride, et autres terres nouvellement par eulx descouvertes. ${ }^{14}$

Le Roy dégage ainsi un principe d'interdépendance, qui rend chaque pays solidaire de tous les autres pays du monde. Les relations des Turcs avec leurs voisins rejaillissent sur la politique européenne de même que c'est en Floride que se joue la rivalité entre le Portugal et la France. Rien n'indique que l'auteur perçoive une solidarité particulière entre les pays d'Europe. S'il les évoque en plus grand nombre, c'est pour deux raisons très évidentes: d'une part, les relations, pacifiques ou belliqueuses, sont évidemment plus apparentes entre pays voisins; et d'autre part, Le Roy est mieux informé de la politique européenne que de l'asiatique ou l'américaine. Si on tient compte de ces deux données, il apparaît que Le Roy décrit l'enchevêtrement des relations internationales au niveau mondial, et qu'il ne cherche nullement à circonscrire un niveau européen. Telle est bien sa conclusion:

En telles manieres les affaires du monde liez et correspondents ensemble, ne peuvent estre bien entendus les uns sans les autres, ny comprins en histoire parfaitte. ${ }^{15}$

En revenant à l'impossibilité de rendre compte en "histoire parfaitte" de relations internationales aussi interdépendantes au niveau mondial, Le Roy retrouve son point de départ: l'impossibilité d'écrire une chronique nationale, et la nécessité de dépasser "l'histoire française" pour esquisser une "histoire universelle".

Le changement de paradigme historiographique qu'opère Le Roy repose sur des croyances astrologiques très répandues au $\mathrm{XVI}^{\mathrm{e}} \mathrm{s}$., qui affirment une relation entre le cours des astres et celui de l'histoire, entre les révolutions

\footnotetext{
${ }^{14}$ Ibid., f. $7 \mathrm{v}-8 \mathrm{r}$.

${ }^{15}$ Ibid., f. 8 v.
} 
du ciel et celles qui affectent la vie politique des Empires ${ }^{16}$. Pour expliquer la "lyaison et correspondance generale de l'Europe, Asie, Afrique, et des Terres neuves" ${ }^{17}$, Le Roy invoque donc les effets combinés de la providence divine et de l'influence des astres, origine unique de tous les événements, mais qui se diversifie selon les temps, les lieux et les circonstances ${ }^{18}$ : les événements terrestres, dans leur variété et leur bigarrure, apparaissent alors comme les conséquences diversifiées d'une même cause céleste. En conséquence, pour écrire l'histoire universelle et saisir l'interdépendance de tous les événements terrestres, Le Roy rêve de s'élever jusqu'à un point de vue céleste, d'où leur unité pourrait se révéler.

Et pour en avoir meilleure cognoissance, je me soubhaitterois maintenant estre eslevé en quelque lieu treshaut et treseminent, d'où je peusse contempler sans passion la condition presente du monde inferieur, et toute la face de la terre habitable [...]. Considerant maintenant les Chrestiens divisez en sectes, fleurissans neantmoins és lettres et és arts. Maintenant les Turcs belliqueux dominans en la meilleure partie du monde, et les vaillans Perses leurs adversaires. Maintenant les Moscovites effrayans le Septentrion et l'Orient par le succez de leurs victoires. Les Tartares et Alarbes perpetuellement vagabons et errans par grosses trouppes comme Citez: les Sauvages du Bresil s'entremangeans: les ingenieux Chines et Cathains se preferans à tous en exquis ouvrages, en richesse et puissance: les Castillans et Portugallois naviguans par tout I'Ocean. Je verrois tout le monde descouvert, dont une grande partie estoit demeurée incongneuë jusques à present: les estremitez d'Orient et Occident, du Midy et du North communiquer ensemble. ${ }^{19}$

Le projet d'Histoire universelle suppose d'embrasser la totalité du monde d'un seul regard. La tendance à l'énumération qui caractérise l'écriture de Louis

\footnotetext{
${ }^{16}$ Voir, par ex., E. Garin, Le Zodiaque de la vie. Polémique antiastrologiques à la Renaissance, Paris, les Belles Lettres, 1991, en part. ch. 1.

17 Le Roy, Considerations sur l'histoire..., f. 8 v.

18 "Mais convient estimer avec quelques sages anciens, tout ce monde inférieur obei: au superieur, et par luy estre gouverné: et qu'estant le premier mouvement au premier ciel, tous les autres en dependent: tellement que selon les aspectz, oppositions, conjonctions, distances, apparences, cachemens des astres, descendent ça-bas diverses influences, rendans les hommes plus disposez en l'une saison qu'en l'autre à la vertu, aux lettres, et aux armes: puis sortans de la mesme cause celeste grans et espouvantables evenemens de guerres, famines, pestes, inondations, tremblemens de terre, seicheresses, et bruslemens alterer tous cas humains par certaines revolutions de l'univers, selon que les parties du ciel et de la terre correspondent, et que la matiere y est disposee. [...] Mais recognoissans par dessus les causes natureles la divine providence gouverner ce monde, nous croions qu'elle despart les graces non mesmes à tous hommes ensemble, mais à chacune nation la sienne et à sa fois, à fin qu'elles n'en enorgueillissent, et se mescognoissent: ainsi les armes, les lettres et les arts s'entresuivans, aller de païs en paiis, et estre plus cultivez en une saison qu'en l'autre" (Ibid., p. $9 \mathrm{v}-10 \mathrm{v}$ ).

${ }^{19}$ Ibid., p. $10 \mathrm{v}-11 \mathrm{r}$.
} 
le Roy correspond au désir de prendre en considération tous les peuples, même les plus éloignés et les moins connus: son attention déborde largement l'Europe, jusqu'à la Chine et au Nouveau Monde. On peut aussi remarquer qu'il porte une appréciation favorable sur la puissance de chaque peuple qu'il prend en considération: nul européocentrisme donc, mais plutôt la conscience éblouie de la diversité et de la richesse de l'humanité, en quelque part que vivent ses représentants.

Malgré la hauteur de vue à laquelle il aspire, Le Roy ne perd pas de vue le royaume de France, et c'est, d'une certaine manière, pour mieux en faire l'éloge qu'il cherche à redéfinir les cadres usuels de l'historiographie. Son opuscule est en effet daté de juin 1567, c'est-à-dire qu'il est écrit au cours d'une période de quelques années de paix, après les premières guerres de religion qui ont ravagé la France. Dans ce contexte où on pouvait espérer la paix durable, l'opuscule de le Roy vise à décharger Catherine de Médicis, reine de France et régente du royaume pendant la minorité de Charles IX, de la responsabilité des premières guerres de religion: on ne peut lui reprocher les turbulences qu'a connues le royaume, puisque celles-ci ne sont pas le fait d'une mauvaise politique, mais d'une disposition astrale néfaste, qui a suscité des crises dans le monde entier. Après avoir rappelé les malheurs subis par la France dans un passé récent, Le Roy poursuit:

Lesquelz maux jaçoit qu'ilz aient esté terribles: toutesfois y aura moindre occasion de s'en esbahir, en considerant la condition universelle de ce siecle merveilleux. Car qui est celuy entre tous les hommes Chrestiens et Barbares qui n'aye beaucoup souffert en ce temps ? [...] Quelle sorte de malheur pourroit on imaginer ou penser, qui ne soit advenue en cest aage ? $^{20}$

Au contraire, ces troubles révèlent bien plutôt l'action bénéfique de la reine, qui a permis à ses sujets de sortir d'une crise qui aurait pu renverser le royaume:

Et seroit le mal allé plus avant sans le soin extreme et diligence qu'avez mise à l'appaiser, vous presentant volontairement à tous dangers, jusques à oublier vostre santé, et quelquefois vous mettre en chemin malade, pour restituer la concorde et tranquillité publique. ${ }^{21}$

On ne peut réduire cet éloge de la reine à une simple flatterie courtisane, car il participe d'une exaltation du temps présent, de portée beaucoup plus

\footnotetext{
${ }^{20}$ Ibid. $\mathrm{f}^{\circ} 3 \mathrm{v}$.

${ }^{21}$ Ibid. f. 3 r.
} 
générale. Le contexte politique troublé sert de révélateur à la vertu supérieure de Catherine de Médicis. C'est un même principe de compensation entre les biens et les maux qui soutient l'appréciation de son époque par Le Roy. Malgré la somme considérable des calamités récentes, l'équilibre des biens et des maux reste favorable, le temps présent surpasse le passé en mal comme en bien, et finalement les réalisations hautes l'emportent.

Toutefois si balançons le mal avec le bien, ne trouverons point y avoir eu par le passé siecle, où les entendemens et les arts liberaux et mecaniques soient parvenus à plus grande perfection que maintenant ${ }^{22}[\ldots]$. Car depuis cent ans ne sont seulement venues en evidence les choses qui estoient auparavant couvertes par les tenebres d'ignorance: mais aussi plusieurs autres cogneues, qui avoient esté entierment ignorees des anciens: nouvelles mers, nouvelles terres; nouvelles façons d'hommes, meurs, loix, coustumes ${ }^{23}[\ldots]$ Ce considéré, devons plus modérément supporter les calamitez survenues, et qui surviennent en cest aage: attendus qu'où sont les excellentes vertus, y viennent ordinairement les grands vices pour les rendre plus illustres: et parmy les excellentes merveilles, naissent souvent les monstres horribles. $^{24}$

Quand il énumère les connaissances nouvelles caractéristiques de son époque, Le Roy évoque en premier lieu les grandes découvertes. Suivront le canon, l'imprimerie et la boussole, mais ce premier moment témoigne que, dans son estimation des biens pas plus que dans son analyse des relations internationales, Le Roy ne borne son attention à l'Europe.

Ainsi, en partant d'une attention spécifique accordée à la France, à ses malheurs comme à sa grandeur, Le Roy découvre la nécessité d'élargir le champ de son attention, et d'abandonner le paradigme d'une histoire nationale pour examiner la complexité synchronique des relations mondiales. Dans le cadre d'une réflexion sur l'Europe, le trait le plus frappant de son entreprise réside dans le fait qu'il ne distingue aucune médiation entre l'histoire française et l'histoire universelle: entre les deux, il n'y a pas de place pour la définition d'une Europe comme échelon politique, et moins encore pour une conscience européenne. Cette absence est d'autant plus remarquable que l'entreprise de Le Roy compte parmi les plus novatrices de son temps: son esquisse d'"histoire universelle" s'inscrit dans la mouvance fraîchement ouverte par Jean Bodin dans sa Methodus ad facilem historiarum cognitionem (Paris, Martin Jeune, $1566)^{25}$, ce Bodin chez qui Lucien Febvre était déçu de ne pas trouver de prise

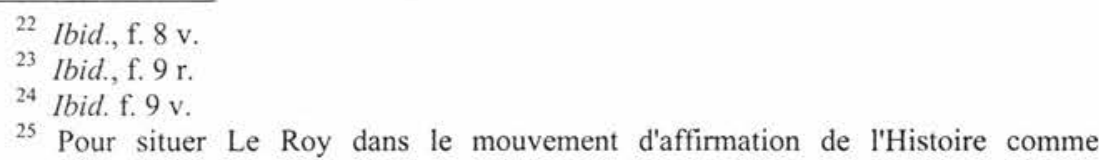


en compte de l'Europe... L'exemple de Le Roy permet de comprendre que cette absence n'est pas un accident, et que même pour les esprits qui ont senti la nécessité d'analyser la politique dans un cadre international et non plus national, l'idée d'Europe n'avait encore aucune consistance. Déborder le cadre du royaume revenait à envisager le monde, pas le continent.

Le traité De la Vicissitude ou varieté des choses en l'univers, publié à Paris, chez Pierre L'huillier, en 1575, développe le projet d'histoire universelle qu'esquissait l'opuscule de 1568. Après un bref exposé sur l'œuvre de la providence, qui règle le monde physique par changements alternatifs, Le Roy se tourne vers l'histoire universelle, où il cherche à mettre ces principes en évidence par l'examen des civilisations successives, chacune d'elles s'élevant jusqu'à un point d'apogée avant de chuter, la providence mesurant ses faveurs, et les distribuant alternativement en différents points du monde. Le traité De la Vicissitude consiste alors en un immense examen comparé des différentes civilisations jusqu'à nos jours. Comme en 1568, Le Roy célèbre son époque audessus des plus grandes civilisations du passé, et il consacre le livre $\mathrm{X}$ à l'éloge "De la puissance, sçavoir, et autre excellence de ce siecle". Il n'accorde aucun privilège a priori à l'Europe, mais prête attention

non aux excellences particulieres des pays: ains entierement aux choses memorables faictes ou advenues durant cest espace de temps par l'Europe, Asie, Afrique, Terres neuves, en Orient, Occident, Septentrion, Midy: et aux graces telles qu'il a pleu à Dieu despartir aux personnes signalees en mesme saison par les contrees diverses de la terre habitable. ${ }^{26}$

C'est seulement par une considération synchronique du monde entier que l'histoire universelle peut espérer dégager les principes actifs de la providence, et un tel projet ne permet pas d'accorder un statut particulier à l'Europe. En conséquence, il commence son éloge de l'époque contemporaine par une évocation admirative de la puissance militaire de Tamerlan, qu'il tient pour le plus grand guerrier de tous les temps. Sa seule faiblesse a été de ne pas avoir un

\footnotetext{
discipline, indépendante des lettres, et pour la relation de son entreprise avec celle de Bodin, voir G. Huppert, L'idée de l'histoire parfaite, Paris Flammarion, 1973, ch. 5 et 6 . Voir également J. Jehasse, "Louis le Roy, maître et émule de Jean Bodin", dans Etudes sur Etienne Dolet, le théâtre au XVIe s., le Forez et l'histoire du livre (mélanges Longeon), Genève, Droz, 1993, p. 251-265 et Ph. Desan, "La Philosophie de l'histoire de Louis le Roy", dans Penser l'histoire à la Renaissance, Orléans, Pardigme, 1993, p. 171-201.

${ }_{26}^{26}$ L. Le Roy, De la Vicissitue ou varieté des choses en l'univers, Paris, Fayard, 1988, p. 364 .
} 
historien à la mesure de son génie militaire ${ }^{27}$. Sur cette note qui limite la puissance ottomane, Le Roy poursuit son examen:

Durant le regne de Tamberlan, commença la restitution des langues et de toutes disciplines. Le premier qui s'appliqua à celle œuvre fut Francisque Petrarque ouvrant les librairies pieça fermees, et ostant la pouldre et ordure de dessus les bons livres des autheurs anciens. ${ }^{28}$

Après les premiers humanistes italiens, Le Roy évoque l'arrivée des byzantins à Florence et la redécouverte du grec, puis les premiers hellénistes italiens, puis, en une très longue liste de plusieurs pages, suivront les grands humanistes français, flamands, allemands, anglais, ecossais, polonais et espagnols; puis après cette énumération par pays, il entreprend une nouvelle liste de grands hommes par domaines d'excellence: d'abord les grands capitaines, au premier rang desquels apparaît encore Tamerlan suivi, entre autres, par Charles VIII ou Charles-Quint; suivent les philosophes, les orateurs, les poètes, les historiens, les jurisconsultes, les mathématiciens, les peintres, les statuaires et graveurs, les architectes, les philologues, les voyageurs, et enfin les princes. Bref, c'est un tableau de ce que nous appelons la Renaissance et que lui appelle "la restitution des bonnes lettres et de toutes disciplines" que déploie Le Roy, et dans cette évocation de la grandeur intellectuelle reconquise par son époque, il n'évoque que des noms européens. Bref, son éloge du présent aboutit au constat d'un partage, d'un répartition des dons que la Providence accorde aux différentes parties du monde: à l'Asie l'excellence militaire; à l'Europe, l'excellence du savoir.

S'il apparaît de façon très visible, ce partage reste cependant implicite, et Le Roy n'en construit pas de théorie: la relation qu'il établit entre l'Europe et les lettres n'est pas essentielle, puisqu'elle n'a pas toujours été réalisée, mais conjoncturelle; elle ressortit à une distribution temporaire des bienfaits de la providence. On voit sans doute poindre ainsi la conscience d'une identité culturelle, mais pour qu'elle prenne véritablement consistance, il faudrait que Le Roy estime permanente la conjonction fortuite entre un espace géographique et la culture littéraire; qu'il sorte du constat, nécessairement relatif à une situation et à un moment particulier, pour affirmer le lien constant entre deux principes; qu'il considère le livre comme un attribut de l'Europe. Or, il ne peut franchir ce

27 "Fortune l'ayant tousjours favorisé, sans jamais luy estre contraire, semble entre tant de succez admirables qui excedent l'ordinaire des conquerans, luy avoir denié un historien excellent de doctrine, et eloquence pareille à ses vertus: à fin de le celebrer dignement", ibid. p. 367.

28 Ibid. 
pas, puisque tout son ouvrage analyse précisément les vicissitudes de la civilisation et de sa distribution, nécessairement instable, dans l'espace et le temps. D'un certain point de vue, cette entreprise constitue un avatar complexe du schéma historiographique de la translatio imperii et studii, et pas plus que lui, elle ne permet de définir des différences qualitatives entre les civilisations: il n'y a pas de culture européenne ou autre, car la civilisation est essentiellement une, la même partout et en tout temps, mais elle se déplace d'un lieu à l'autre. L'histoire universelle ne s'accommode pas d'une approche ethnographique: conçue comme l'analyse des distributions variables des bienfaits de la providence, elle postule une unicité de la culture, dont les réalisations ne se distinguent que par la hauteur atteinte par ceux qui la poursuivent.

On mesure ainsi quelle résistance certains des courants de pensée les plus novateurs de la fin du XVIe siècle pouvaient opposer à l'émergence d'une conscience européenne. Pour saisir les prémisses, encore incertaines, de celleci, il faut se détourner des discours politiques, de ces discours directement en prise avec les institutions humaines comme celui du mémorialiste ou de l'historien, pour envisager la représentation poétique, où l'Europe commence alors à recevoir une personnalité et des valeurs, à la faveur d'un processus de personnification de la notion géographique.

Les Epithetes de Maurice de la Porte (Paris, G. Buon, 1571) permet de dégager les valeurs les plus couramment associées à l'Europe. Il s'agit d'un dictionnaire poétique, qui répertorie, par ordre alphabétique, les substantifs noms propres et communs - dont les poètes font fréquemment usage. Chaque nom est suivi d'une série d'adjectifs (les "épithètes" du titre) qui peuvent lui être associés et, éventuellement, d'une courte glose. Dictionnaire des lieux communs littéraires, l'ouvrage ne prétend nullement transmettre un savoir nouveau ou original; il constitue bien plutôt un inventaire des représentations ordinaires que pouvait avoir un lettré, et c'est à ce titre qu'il nous intéresse: il permet de dégager la conception la plus commune de l'Europe. A ce mot, on peut donc lire:

Europe, Riche, féconde, agenoride, populeuse, belle, phenicienne, excellente, fructueuse, temperée, salubre.

Europe ainsi appellée d'Europe fille d'Agenor roi des Pheniciens, est la premiere et moindre partie du monde, mais trespopuleuse, tresfertile, et bien cultivée. N'aiant aucune region où les hommes ne se soient fait habitation, et où ils ne se puissent commodement procurer les choses qui sont necessaires pour la vie. Elle est close vers l'Occident par la mer Atlantique vers les colomnes d'Hercule, au nord et septentrion, elle a la mer Angloise, et tout le trait de Norvege et mer glaciale: Au levant son limite est borné par le fleuve Tanaï, Paluz Meotides et mer majour: et au midi la mer mediterrannée la separe de l'Afrique. 
D'un point de vue géographique, l'Europe se définit par ses contours: c'est une zone sans caractérisation interne, mais la notice déborde très vite la géographie pour identifier la "partie du monde" au personnage mythologique d'Europe. Aux épithètes qui renvoient au continent ("riche, feconde... populeuse") se mêlent celles qui renvoient à la fille d'Agenor, roi des Phéniciens, évoquée dans la fable ("agenoride" ou "phenicienne"). Sans doute cette présence mythologique s'explique-t-elle par la dimension poétique de l'ouvrage: La Porte travaille à partir de l'œuvre des poètes, et il écrit pour les poètes; or, à la Renaissance, l'usage de fables antiques est un des caractères fondamentaux de l'écriture poétique. Il n'en reste pas moins qu'une telle superposition entre notion géographie et figure mythologie révèle à la fois une conscience extrêmement floue de l'Europe dans sa définition géographique ${ }^{29}$, et la tentation d'en saisir l'unité par le biais de la représentation humaine. On trouve une démarche analogue dans certaines séries de gravures représentant les continents où, suivant une tradition ancienne, encore vivace au XVI ${ }^{\mathrm{e}}$ s., l'Europe est représentée sous l'apparence de l'héroïne mythologique du même nom: une jeune femme, assise sur le dos d'un taureau courant sur la mer.

Dans d'autres images, la personnification allégorique se détache de la figure mythologique, et de ce fait se précise. L'Europe est représentée comme une femme, entourée d'attributs symboliques, permettant de l'identifier, et qui constituent autant d'indices visuels des connotations attachées à la notion d'Europe. La représentation allégorique oblige en somme à définir la personnalité symbolique de l'Europe, et à la doter d'une identité. Examinons par exemple une série de quatre gravures d'Adrien Collaert, réalisée à partir de dessins que Marteen de Vos a réalisés pour l'entrée d'Ernest d'Autriche à Anvers en 1594, représentant les quatre parties du monde (ill. 1 à 4 ; on notera la présence de l'Amérique). Sans doute la figure d'Europe montée sur son taureau a-t-elle servi de prototype à cette série, où les continents sont représentés comme des fem-

${ }^{29}$ On peut discerner un même flottement si on compare l'entrée "Europe" à celle que M. de la Porte consacre à l'Afrique et à l'Asie (l'Amérique n'est pas évoquée, preuve, s'il en était besoin, de l'écart qui peut exister entre l'état réel des connaissances géographiques, et les représentations du monde: La Porte ne nous donne accès qu'aux secondes). Si l'Afrique se distingue des deux autres continents en ce qu'elle est jugée hostile à la vie humaine(voici la liste des épithètes accolées à l'Afrique: "Affrique. Sablonneuse, alterée, monstrueuse, fertile, vague, ardente, nourriciere de monstres, inhabitable, spatieuse"), l'Europe et l'Asie partagent les caractère de grandeur et de puissance (voici la liste des épithètes accolées à I'Asie: "Asie. Fertile, puisant, riche, grande, spatieuse"). Tout juste peut-on percevoir que l'Europe, qualifiée de "tempérée" ou de "salubre", est plus hospitalière à ses habitants. L'Europe n'évoque ni une entité culturelle, ni une entité politique: c'est une partie du monde, la seule bien souvent qu'un auteur connaisse, qui se caractérise conventionnellement par la douceur des ses conditions naturelles, et qui, de ce fait, mérite plus d'éloge que les autres. 
mes assises sur des animaux, mais c'est paradoxalement la figure d'Europe qui s'écarte le plus de ce modèle, car elle n'est pas assise sur un animal: elle est assise, en tenue d'impératrice, sur une boule représentant la terre. Cette iconographie est glosée par le poème latin placé sous l'image:

Induperatrici decorata EUROPA corona

Orbem quo sedet ut solio regina superbo,

Cum numero populi haud posset superare vel auro

Viribus et valido sibi subditi inclyta ferro.

Europe, coiffée d'une couronne impériale; / Le monde, sur lequel elle est siège telle une reine sur son trône orgueilleux, / Comme elle ne pouvait le surpaser en abondance de peuple ou en or,/ elle l'a soumis par ses hommes et par le fer vigoureux.

Ces vers placent l'Europe et le monde dans un rapport de concurrence, qui dégénère d'une saine émulation en opération de conquête et de colonisation. Avec une confiance déconcertante dans la légitimité de l'Europe à revendiquer une hégémonie mondiale, cette image présente l'impérialisme militaire comme un autre moyen d'assurer à l'Europe la place d'impératrice qui lui revient. Cette iconographie n'a rien d'exceptionnel: on la retrouve sous une forme très similaire dans l'Iconographie, de Cesare Ripa. Cet ouvrage répertorie et décrit les principales figures allégoriques qu'un peintre peut utiliser. Publié en 1593 sans images, il est réédité illustré à Rome en 1603 et traduit en français par Jean Baudoin en 1643: cet ouvrage a connu un très gros succès, et il a influencé l'art du siècle suivant. L'Europe y apparaît comme une femme couronnée, entourée de symboles de l'abondance et des arts (ill. 5). Le commentaire souligne sa fertilité, sa piété, sa puissance, mais tous ces caractères sont subordonnés, comme autant de spécifications, à son caractère royal. La suprématie européenne constitue le premier trait que le commentaire met en avant, et celui qu'il attache plus particulièrement au symbole de la couronne

Cette partie du Monde qui excelle par dessus toutes les autres, nous est figurée par une Dame royallement vestuë d'une robbe de plusieurs couleurs. Elle porte sur la teste une riche Couronne, et se voit assise au milieu de deux Cornes d'Abondance [...]

La Couronne qu'elle porte fait voir que l'Europe a toûjours eu le principal advantage sur les autres partie de l'Univers dont elle a triomphé comme Reine ${ }^{30}$.

L'Europe dominatrice du monde, impératrice ou reine, constitue la première figure moderne de l'Europe.

${ }^{30}$ C. Ripa, Iconologie, éd. et trad. Jacques Baudoin, Paris, 1643 (fac simile: Paris, Aux amateurs de livres, 1989), seconde partie, p. 8-9 
La conclusion est la même si on examine un autre type de personnification de l'Europe. Il s'agit d'un tracé anthropomorphe de la carte du continent, où l'Europe apparaît comme une impératrice: l'Espagne constitue la tête; la péninsule italienne, le bras droit; le Danemark, le bras gauche. Inventée en 1537 par Johann Putsch, cette allégorie a connu plusieurs avatars au cours du seizième siècle, dont nous reproduisons le plus connu (ill. 6), qui apparaît dans les éditions de la Cosmographie, de Sebastian Münster ( $1^{\mathrm{e}}$ éd. 1544) à partir de $1588^{31}$. Le sens de cette gravure tient moins à la figuration anthropomorphe du continent, qui ne fait qu'exploiter, en les sollicitant quand même assez fortement, les suggestions de la géographie, mais aux attributs impériaux - globe, couronne et sceptre - qui, eux, ne sont aucunement motivés par la géographie, et dont la présence ne s'explique que par une décision du dessinateur. Il s'agit moins d'affirmer l'unité organique de l'Europe, qui constituerait un seul corps, que sa puissance. La figuration anthropomorphe n'est que la conséquence du parti impérial: il faut bien donner un corps à cette impératrice symbolique. Mais où sont les sujets de cette souveraine ? Absents de l'image, ils ne peuvent se trouver que dans le reste du monde connu: Afrique, Asie et, peut-être, Amérique. Si d'ailleurs l'Europe est figurée, non en reine, mais en impératrice, c'est bien pour indiquer sa vocation à la domination mondiale. Peut-on pousser la lecture politique plus loin, en constatant que l'Espagne se trouve littéralement à la tête de cet empire européen ? Sans doute, encore qu'on voie mal où placer ailleurs qu'en Espagne, la tête de l'Europe. Quoi qu'il en soit, cette lecture proespagnole de l'allégorie ne se fonde pas sur la rivalité perpétuelle de CharlesQuint avec la France: celle-ci nous renverrait en effet à un problème intérieur à l'Europe, et le traitement de la géographie est si approximatif qu'à l'évidence, l'auteur de l'allégorie ne s'est guère soucié de l'organisation interne de l'Europe. Il faut plutôt songer au rôle de l'Espagne et du Portugal dans les grandes découvertes. La devise de Charles-Quint consistait d'ailleurs en deux colonnes, qui figuraient le détroit de Gibraltar connu alors sous le nom des "colonnes d'Hercule", accompagnées du mot "plus oultre": s'affirmait ainsi l'ambition de l'Espagne à étendre sa puissance au-delà de l'Europe, vers l'Afrique d'abord, mais aussi dans toutes les directions, au-delà du monde connu. L'impératrice Europe reflète la même idéologie d'une hégémonie mondiale. Dans cette perspective, il est significatif que ce soit dans des séries consacrées aux continents que nous ayons vu se préciser la personnalité allégorique de l'Europe: c'est par

${ }^{31}$ Sur cette figure, voir S. Deswarte-Rosa, "Le Portugal et la Méditerranée. Histoires mythiques et images cartographiques", Arquivos do Centro Cultural Calouste Gulbenkian, XLIII, Lisbonne-Paris, Centro Cultural Calouste Gulbenkian, 2002, p. 97-147, en part. p. $130-135$. 
rapport aux autres parties du monde, sur lesquelles elle affirme sa domination, que l'Europe se définit.

Cette aspiration à une hégémonie mondiale marque la réinterprétation et le dépassement de l'idéologie impériale qui a eu cours pendant tout le Moyen$\hat{A}_{\mathrm{ge}}{ }^{32}$. En tant que réalité politique, l'Empire disparaît avec la chute de Rome, pour ne réapparaître qu'en 800 , avec le couronnement impérial de Charlemagne. Ce nouvel empire aura une existence effective brève, même si les provinces allemandes continueront à élire l'empereur du Saint Empire Romain Germanique pendant des siècles. L'importance de l'Empire carolingien tient surtout à ce qu'il a réintroduit l'idée impériale dans l'idéologie politique européenne, et elle n'en sortira plus. Du Moyen-Âge jusqu'au XVII ${ }^{\mathrm{e}}$ s. au moins, cet idéal s'affirme dans les domaines politiques et religieux: il s'agit tout à la fois de ramener le monde sous une domination unique, et de le ramener à l'unité de la foi chrétienne. Les croisades participent de cette ambition. Cette idéologie se double souvent de perspectives eschatologiques plus ou moins prononcées, diverses théories affirmant que l'avènement d'un empereur universel précèderait de peu le Jugement dernier. $A u X V I^{\mathrm{e}}$ s., cet idéal reste fort: Charles-Quint est élu empereur du Saint-Empire contre François I ${ }^{\mathrm{er}}$, et l'étendue de ses territoires laisse envisager la possibilité d'une réalisation politique de son titre. Pendant les $\mathrm{XVI}^{\mathrm{e}}$ et $\mathrm{XVII}^{\mathrm{e}}$ siècles, les propagandes françaises et espagnoles développent des théories impériales au profit de leurs souverains respectifs. Cependant, les réformes et l'affirmation des États en Europe tendent progressivement à périmer l'idée d'une unification religieuse et politique de la Chrétienté, tandis que les grandes découvertes ouvrent largement l'espace du monde à conquérir. L'idée impériale médiévale cède progressivement à l'impérialisme colonisateur moderne; et c'est précisément à ce moment que la notion d'Europe pénètre le discours politique. C'est aussi le moment où on représente le continent sous une forme humaine: il s'agit moins d'affirmer l'unité (problématique) des pays européens, que les droit d'une Europe figurée en monarque à s'approprier le monde.

Un dernier texte nous permettra de préciser la fonction de l'allégorie, et le lien entre les notions d'Europe et d'Empire: il s'agit d'un texte d'Etienne Jodelle, tiré du Recueil des Inscriptions (Paris, A. Wechel, 1558). Cet ouvrage est le compte-rendu d'une mascarade qu'à la demande de la municipalité pari-

${ }^{32}$ De l'abondante littérature sur l'idée d'Empire; signalons R. Folz, L'idée d'Empire en Occident du $V^{e}$ au XIV siècle, Paris, Aubier-Montaigne, 1953 ; F. Yates, Astrée. le symbolisme impérial au XVIe s., Paris, Belin, 1989 et A. Haràn, Le lys et le globe. Messianisme dynastique et rêve impérial au XVIe et XVIIe s, Paris, Champ Vallon, 2000. 
sienne, le poète a organisée pour accueillir le roi Henri II. Après la très lourde défaite de Saint-Quentin, à l'automne 1557, on a pu craindre que les troupes espagnoles renversent la monarchie française, mais le retour du duc de Guise d'Italie a permis au roi de France de redresser la situation. Dans les premiers jours de 1558 , les troupes françaises réussissent même à reconquérir Calais, forteresse jugée inexpugnable que les Anglais tenaient depuis près de deux siècles: la menace d'une prise en tenaille des Français entre les Anglais et les Espagnols s'éloigne alors et, pour célébrer cette victoire, la municipalité parisienne a voulu accueillir dignement le roi Henri II. Cependant, la mascarade commandée à Jodelle à cette occasion a été trop vite préparée, et elle a été fort mal réalisée. C'est pour se justifier de cet échec que le poète publie le Recueil des inscriptions, qui consiste dans sa première partie, en un livret d'entrée auquel s'entremêle un plaidoyer pro domo. Le poète cherche tout à la fois à restituer la cohérence de son invention, occultée par une réalisation défaillante, et à dégager sa responsabilité de l'échec. C'est toutefois la seconde partie de l'ouvrage qui nous intéresse ici. À la suite de cette apologie, Jodelle insère en effet un recueil d'Icones, type de recueil d'épigrammes qui a connu un certain succès dans la poésie néo-latine du temps ${ }^{33}$. En général, les recueils d'Icones présentent une suite d'épigrammes sur les portraits des grands hommes de l'Antiquité, parmi lesquels se glissent parfois un ou deux éloges de contemporains, mais alors rares, et déjà illustres. Pour la première fois peut-être, Jodelle modernise le genre, en consacrant ses épigrammes de manière exclusive à l'éloge de contemporains, et parmi ceux-ci, il ne retient que des personnalités politiques ou militaires. Le titre de cet opuscule est explicite: "Christianorum nostri temporis Heroum et Heroinarum Icones", "Images des héros et des héroïnes chrétiens de notre temps". Dans une première partie, la plus abondante, il célèbre les personnalités éminentes de la cour de France, et des hommes de guerre occupant une place clé dans le dispositif militaire du roi. Après ces images "des Héros français" ("Gallicorum heroum"), il consacre quelques poèmes à des personnalités étrangères ("externorum aliquot"), grandes figures de la politique européenne contemporaine, à qui Jodelle décerne éloge ou blâme selon qu'elles sont favorables ou hostiles à la France. Il s'agit en somme pour le poète de prouver ses compétences pour la célébration politique, dont l'échec de la mascarade aurait pu conduire à douter.

Pour notre propos, il faut surtout souligner que ce recueil constitue un instantané de la politique européenne, ou ses principaux acteurs se trouvent

${ }^{33}$ Voir P. Laurens, L'abeille dans l'ambre. Célébration de l'épigramme, Paris, Les Belles Lettres, 1989, p. 420-429. 
évoqués un à un. La deuxième partie sur les figures internationales est, en l'état, beaucoup plus maigre que la première, mais le poète se promettait de la développer, pour peu que son livre ait eu du succès. De façon significative, ce recueil s'ouvre sur une image, non plus individuelle mais allégorique, "De l'Europe désolée et déchirée" ("lugentis et lacerce Europa") ${ }^{34}$. Sous ce titre, ce n'est pas un tristique, mais une longue inscription qui développe les paroles qu'est censée prononcer l'Europe personnifiée, représentée sur un tableau que nous ne connaissons plus. Quoi qu'il en soit, cette Europe s'adresse aux passants, et le texte de Jodelle constitue ses paroles. Elle commence par se présenter et rappelle qu'elle a autrefois accueilli deux empires, celui des grecs et celui des romains:

VOSTRA EGO ILLA TERRAR. QVONDAM OMNIVM FORTVNATISS. EVROPA DVAB. OLLIS POTENTISS. GENTIB. GRÆCIS PRIMVM DEINDE ROMAN. IMPERANTIB. VNIVERSI TERRAR. ORBIS BIS DOMINATRIX... ${ }^{35}$

Elle évoque ensuite le troisième règne qu'elle a accueilli: non plus un empire politique comme auparavant, mais la chrétienté dont elle est devenue le foyer.

...NON IAM CORNVTO VT FINGITVR IOVI SED DEO OPT. MAX. RESTITVTÆQ. SALVT. AVTHORI

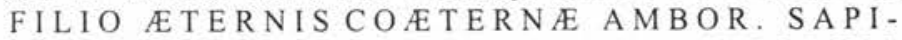
ENTIÆ CONSILIIS DELECTA SANCTIORVM VIRORVM BEATIORVMQ. DÆMONVM MINISTERIIS DEVOTA ET HACTENVS INTER SORORES PATERNIS AMORIB. FOELICITER DILECT A... ${ }^{36}$

\footnotetext{
${ }^{34}$ Voir E. Jodelle, Le recueil des inscriptions, 1558 , éd. V. Graham et W. McAllister Johnson, University of Toronto Press, , 1972, p. 139-141.

${ }^{35}$ trad. 'Moi, votre Europe, autrefois la plus heureuse de toutes les terres ; quand ces deux peuples très puissants, les Grecs d'abord, puis les Romains, avaient l'empire, deux fois maîtresse du rond entier de cette terre"

${ }^{36}$ trad. "et maintenant élue, non par Jupiter, qui est représenté cornu, mais par Dieu très-bon très-grand, par son fils, auteur de notre salut restitué, et par le conseil éternel de la sagesse co-éternelle aux deux ; consacrée par les ministères d'hommes très saints, et d'esprits très heureux ; et jusqu'alors, heureusement favorisée entre mes sœur par l'amour paternel".
} 
Puis elle évoque enfin sa situation en 1558, alors que les guerres qui opposent la France à l'Espagne et l'Angleterre la ravagent.

\author{
...NVNC VERO PROH DEVM IMMOR -
}

TAL. QVOD VIX POTVISSET SPERARIER MA -

TRVM OMNIVM MATER MISERR. VOS HIC

CVM LACHRVMIS ROGO FILII HOSPITES VT

IN LVGENTE LACERA ET PERTVRBATISS.

PARENTE TANQVAM IN NAVFRAGOR. TA-

BVLA VOSTRÆILLIVS TAM EGREGIÆLAV -

DIS TRIVMPHORVMQ. AMPLISSIMOR. FI -

GVRAM SPECTETIS MISERABILEM ${ }^{37}$

L'image de l'Europe se confond donc avec le tableau d'un naufrage, qui se mesure dans la confrontation entre sa splendeur passée et sa misère actuelle. Son désespoir est d'autant plus grand que sa ruine présente succède immédiatement à son apogée, ce moment où l'Europe a été éclairée par le règne de François $\mathrm{I}^{\mathrm{er}}$, et où elle a atteint le sommet de sa gloire et de son prestige culturel. Sans espoir donc, elle lance aux Français cet appel pour le moins effrayant:

TE VERTITE RAPITEGLADIIS IGNEM IGNIB.

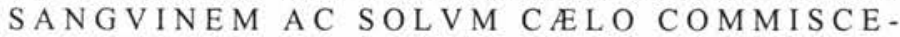

TE CONFVNDITE NEC PRIVS IPSIS CALAMI-

TOSISS. MATRIS VISCERIB. PARCERE LICE-

AT QVAM VOSTRIS FATALIB. VICTORIIS -

AD III ORB. IMPERIVM TANQVAM EX FV-

NERE REVOCATA DE MEIS IPSIVS ET BAR -

BAROR. SPOLIIS TRIVMPHVM AGAM CE-

LEBERR. ${ }^{38}$

Ce texte propose incontestablement une représentation de l'Europe, et il fonde sur cette figure une analyse et un projet politiques; mais de quoi l'Eu-

${ }^{37}$ trad. "mais maintenant, hélas, dieux immortels, à peine aurait-on pu le prévoir, la mère la plus malheureuse de toutes les mères. Fils et passants, avec larmes, je vous demande qu'ici, dans votre parente désolée, déchirée, et bouleversée, ainsi que dans le tableau d'un naufrage, vous contempliez l'image malheureuse de votre gloire si haute et de vos plus grands triomphes".

${ }^{38}$ trad. "agissez, bouleversez, prenez, mêlez le feu avec le fer, le sang avec le feu et la terre avec le ciel, confondez, il ne convient pas d'épargner ces viscères de votre calamiteuse mère avant que, grâce à vos fatales victoires, je sois rappelée, comme du tombeau, vers le troisième empire du monde et que je fasse un triomphe très nombreux avec les dépouilles des miens même et des barbares". 
rope est-elle au juste l'allégorie ? de quelle institution ou de quelle valeur ? Dans les faits, elle ne correspond à rien, puisque les royaumes de France, d'Espagne et d'Angleterre sont en guerre. L'enjeu de l'image est précisément de faire apparaître une unité qui n'existe pas encore. Si la femme porte les stigmates de la guerre dans sa mise dépenaillée et dans son visage affligé, ces signes fonctionnent comme des attributs, qui caractérisent la situation conjoncturelle du personnage, c'est-à-dire qu'ils supposent la présence d'un corps sur lequel ils viennent se greffer comme les signes d'un état temporaire. Or, c'est précisément ce corps nécessaire à la représentation des attributs qui impose l'idée d'une Europe comme totalité organique que la guerre compromet: l'idée d'une unité européenne n'émerge donc qu'à la faveur de l'allégorie, et elle ne coïncide avec aucune organisation politique actuelle, puisque c'est l'enjeu même de l'inscription que d'appeler une structure politique qui concrétiserait l'unité du continent: dans son discours, l'Europe rappelle qu'elle a accueilli les empires grecs et romains - mais ils sont passés -, et souhaite voir advenir un troisième empire, celui des Français - mais il n'est pas encore réalisé. Force est alors de conclure que c'est seulement à une zone géographique que l'allégorie donne forme humaine, à un espace qui a été, et qui de ce fait est appelé à être, le foyer d'une civilisation dominant le monde. Entre l'Europe et l'Empire, l'adéquation n'est pas fortuite: les deux notions supposent une homogénéisation de l'espace, qui renvoie à la continuité géographique des territoires dans le cas de l'Europe, et à la paix qui rend tout lieu librement accessible dans le cas de l'Empire. La représentation de l'Europe sous une forme humaine vise à mettre en relief cette homogénéité spatiale, assimilée à une unité organique, et prépare la projection de la notion dans le domaine politique. L'allégorie joue donc un rôle fondamental dans l'usage politique de la notion d'Europe, et elle détermine son association à l'Empire, idéologie politique qui affiche la visée d'une unité mondiale (de l'une à l'autre, il y a bien sûr cette différence que l'Empire suppose qu'un peuple obtienne l'hégémonie, ce qui implique une phase de guerre victorieuse: par une ruse dont la rhétorique diplomatique est coutumière, l'unité européenne peut ainsi servir, comme chez Jodelle, à justifier les ambitions conquérantes d'une nation, les Français en l'occurrence). En somme, nulle conscience européenne à l'origine de l'Europe, mais seulement une figure: l'histoire ultérieure de l'idée européenne suggère que la politique n'est bien souvent qu'une manière de prendre la rhétorique au mot. 
ILUSTRATIONS

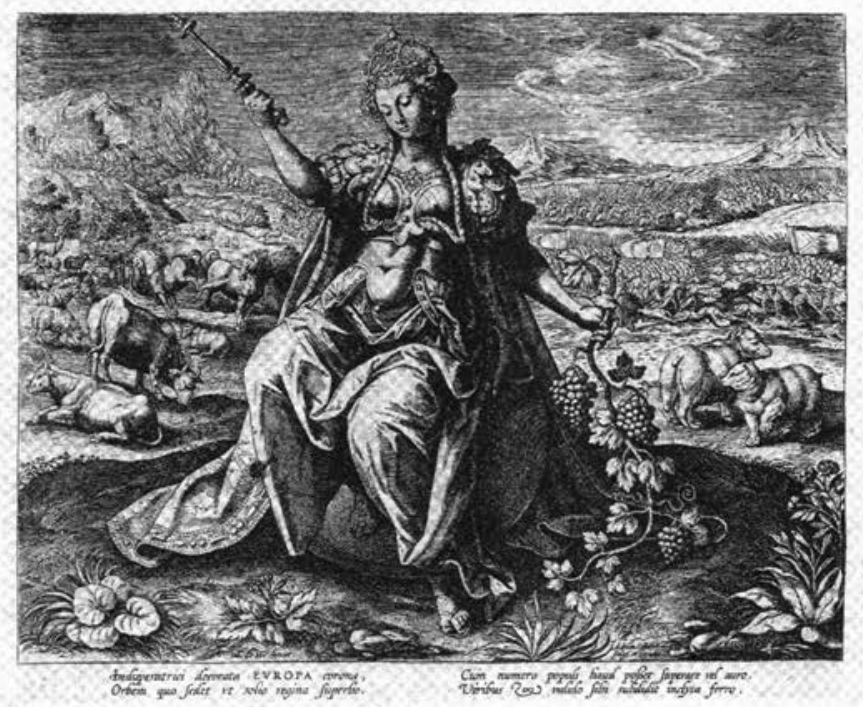

ill. 1 : Adriaen Collaert (d'après Maarteen de Vos), Europe, fin XVI's., gravure sur cuivre.

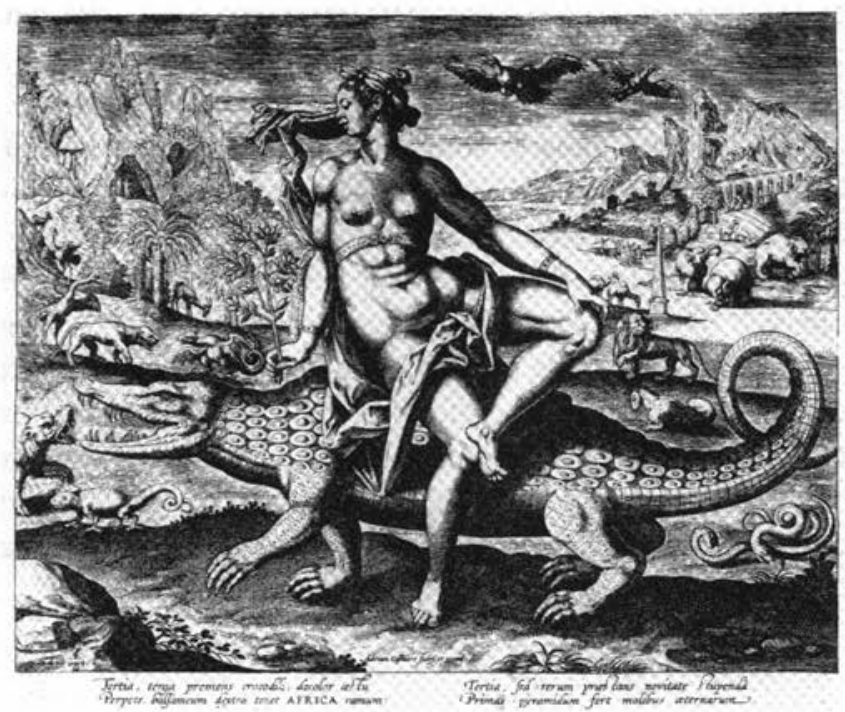

ill. 2 : Adriaen Collaert (d'après Maarteen de Vos), Asie, fin XVI ${ }^{\mathrm{e}}$ s., gravure sur cuivre. 


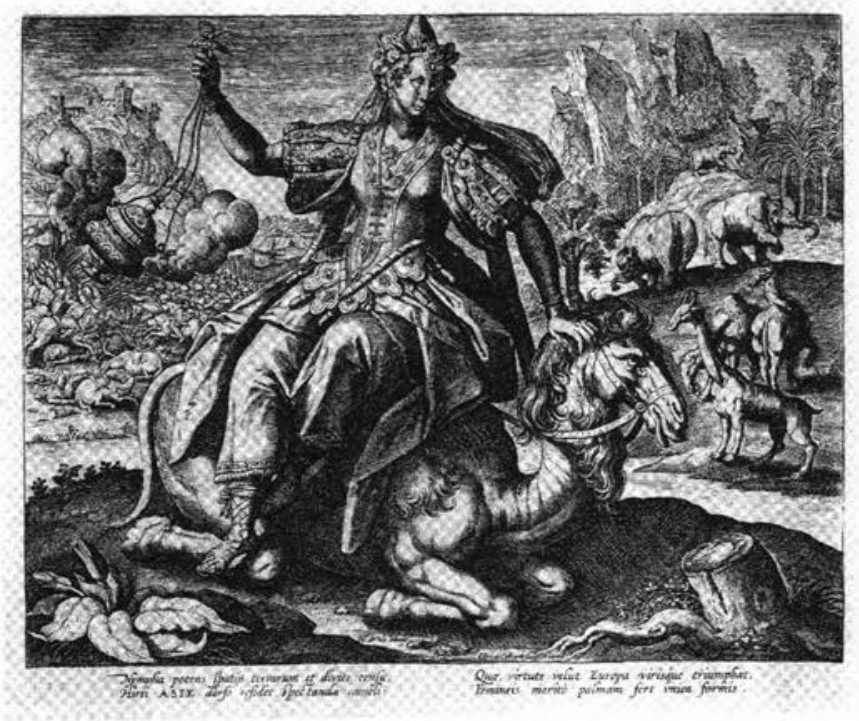

ill. 3 : Adriaen Collaert (d'après Maarteen de Vos), Afrique, fin XVI ${ }^{\mathrm{e}}$ s., gravure sur cuivre.

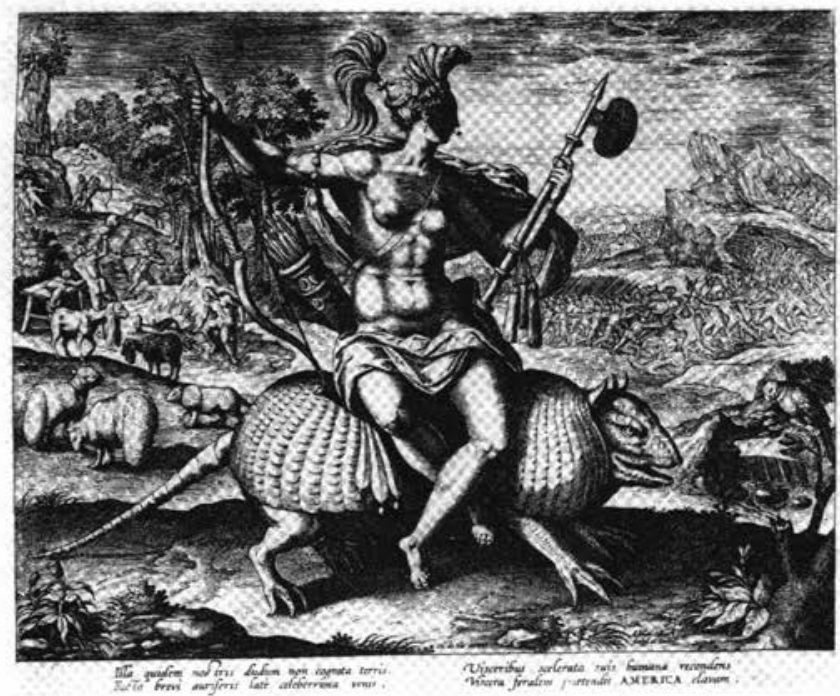

ill. 4 : Adriaen Collaert (d'après Maarteen de Vos), Amérique, fin XVI ${ }^{\mathrm{e}}$ s., gravure sur cuivre. 


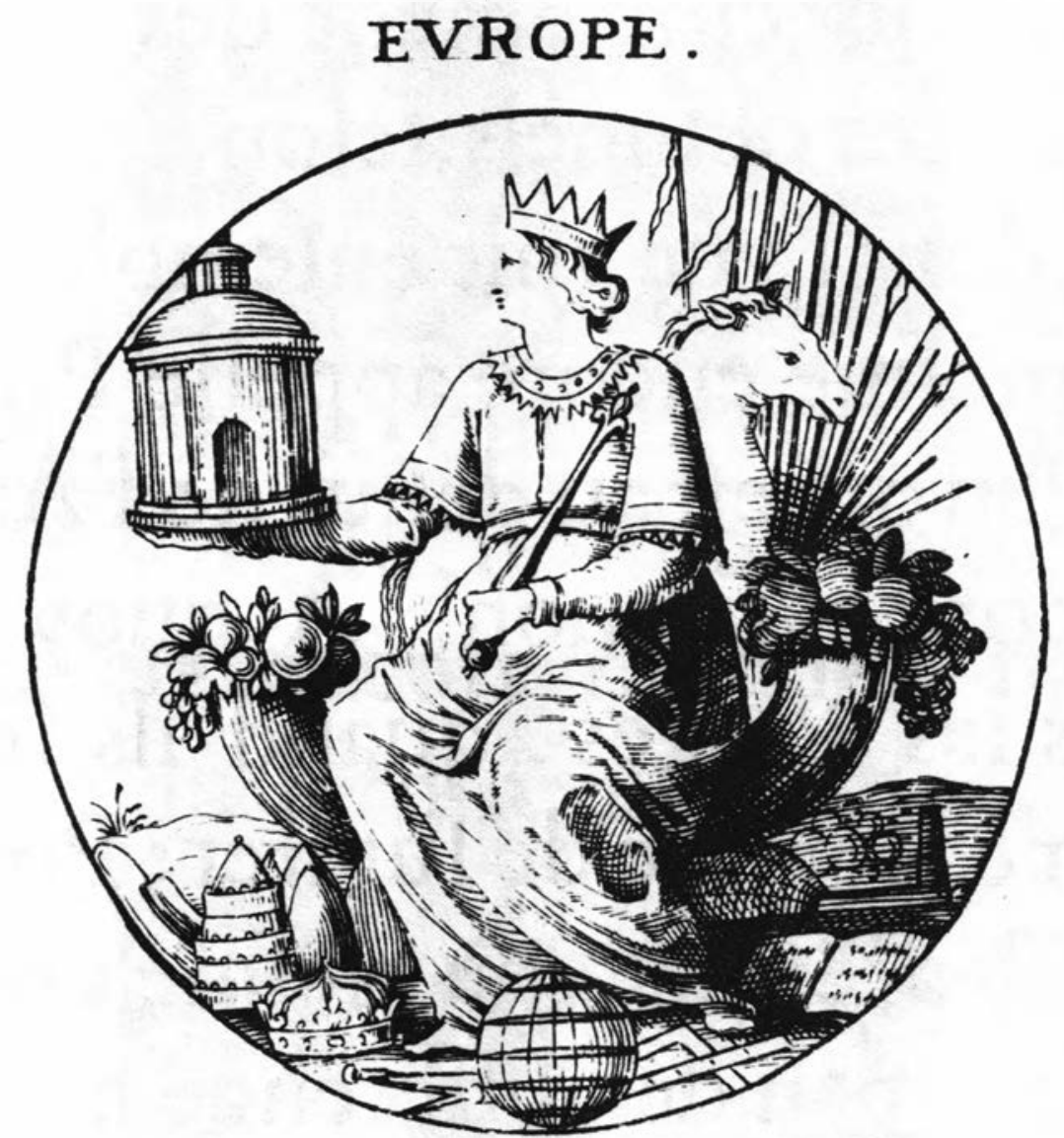

ill. 5 : Jacques de Bie, Europe, gravure sur cuivre tirée de C. Ripa, Iconologie, trad. J. Baudoin, Paris, 1643. 


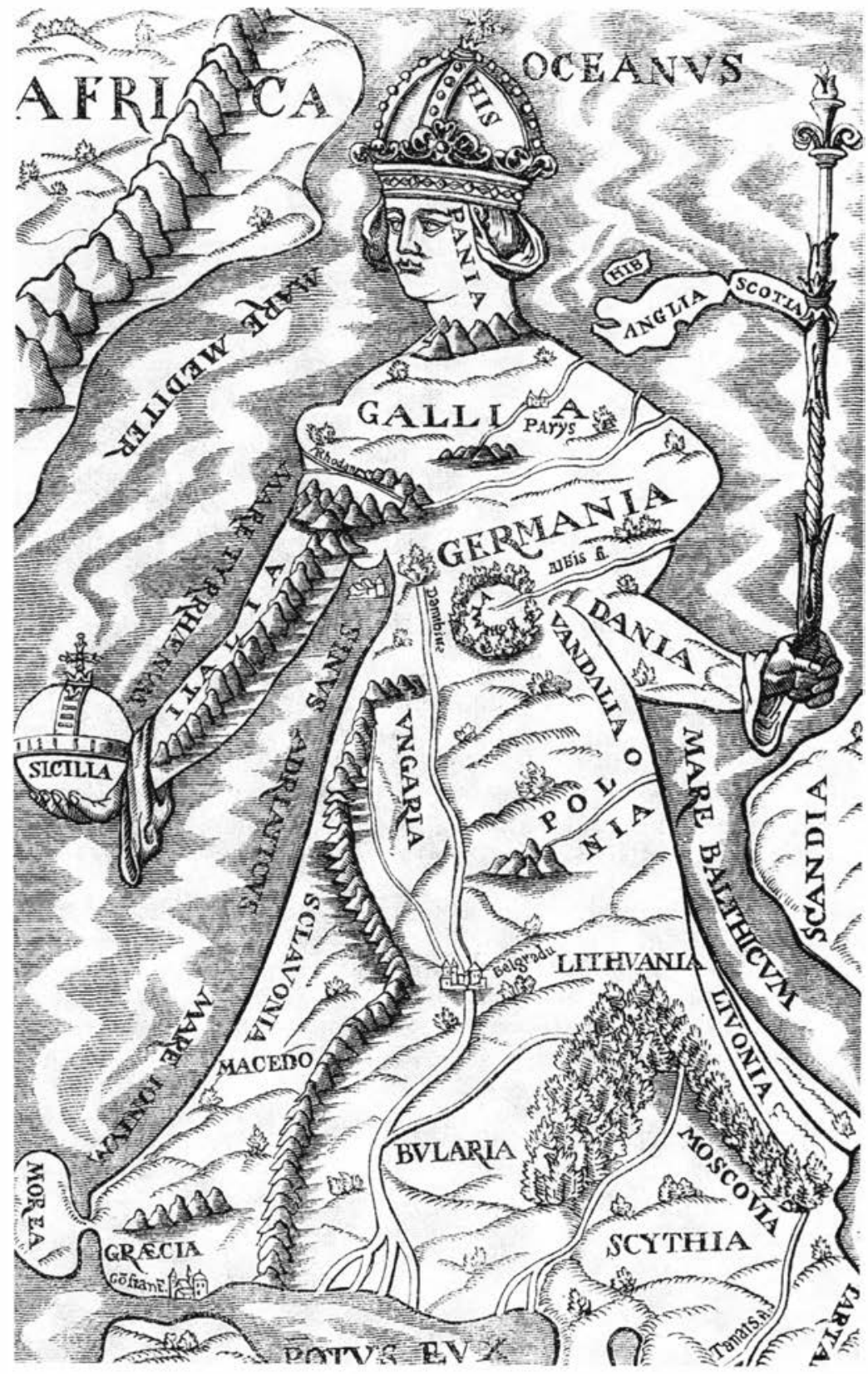

ill. 6 : Europe impératrice, tirée de S. Münster, Cosmographia, 1588. 